\title{
Capsule Commentary on Castro et al., Statin Use, Diabetes Incidence and Overall Mortality in Normoglycemic and Impaired Fasting Glucose Patients
}

\author{
Alice Ching, MD \\ Medical College of Wisconsin, Milwaukee, WI, USA. \\ J Gen Intern Med 31(5):535 \\ DOI: $10.1007 / \mathrm{s} 11606-016-3638-\mathrm{x}$ \\ (c) Society of General Internal Medicine 2016
}

$\mathrm{E}$ very medical decision is a balance between potential benefit and potential risk. Statins have recently been associated with an increased risk of developing diabetes, which has raised concerns given their widespread use. ${ }^{1}$ Castro et al. ${ }^{2}$ investigated the association between statin use and the risk of diabetes and overall mortality in an observational cohort study of over 18,000 non-diabetic patients over a 6year period. The authors found that statins were associated with an increased risk of developing diabetes and a similar reduction in mortality, and conclude that the overall benefit of statins outweigh the incident diabetes risk.

This study has a number of important strengths. The focus on mortality data adds to the information that clinicians need in order to balance risk and benefit. Although the study lacked a matched control group, the authors excluded participants not on medications, as these patients were presumably younger and healthier. Some weaknesses are also noteworthy. As the authors note, data on dosages, fill records, and duration of statin use were unavailable. It is possible that as hyperglycemia progressed in statin-treated patients, physicians discontinued or decreased statin therapy, leading to an attenuation of the observed effect. Importantly, as this was observational data, causal relationships are impossible to prove and should be viewed with skepticism.

This work adds to the growing body of information to help patients and providers make more informed decisions. Other

studies have reported increased diabetes risk over a longer duration, ${ }^{3}$ and long-standing diabetes has been shown to increase the risk of coronary heart disease. ${ }^{4}$ The long-term impact on the incidence of diabetes and mortality remains to be seen.

However, based on the available data, the balance of benefit and risk falls in favor of continuing to prescribe statins according to current guidelines, even in patients with impaired fasting glucose. The authors suggest a reasonable approach with periodic fasting glucose monitoring. Not to be ignored is the impact of lifestyle modification on mortality risk; in this study, the hazard ratio for smoking as a predictor of mortality far exceeds any of the other variables that were included.

Corresponding Author: Alice Ching, MD; Medical College of Wisconsin, Milwaukee, WI, USA (e-mail: alice.ching@va.gov).

\section{Compliance with Ethical Standards:}

Conflict of Interest: The author declares that she does not have a conflict of interest.

\section{REFERENCES}

1. Sattar N, Preiss D, Murray HM, et al. Statins and risk of incident diabetes: a collaborative meta-analysis of randomised statin trials. Lancet. 2010;375(9716):735-742.

2. Castro MR, Simon G, Cha SS, et al. Statin use, diabetes incidence and overall mortality in normoglycemic and impaired fasting glucose patients. J Gen Intern Med. doi: 10.1007/s11606-015-3583-0

3. Macedo AF, Douglas I, Smeeth $\mathbf{L}$, et al. Statins and the risk of type 2 diabetes mellitus: cohort study using the UK clinical practice research datalink. BMC Cardiovasc Disord. 2014; 14:85.

4. Seshasai SR, Kaptoge S, Thompson A, et al. Diabetes mellitus, fasting glucose, and risk of cause-specific death. N Engl J Med. 2011;364:829-841. 